\title{
ALTERAÇÃO NO MÉTODO DE AMOSTRAGEM DE MAHANARVA FIMBRIOLATA (STÅL, 1854) (HEM.: CERCOPIDAE) E AVALIAÇÃO DA EFICIÊNCIA DE METARHIZIUM ANISOPLIAE (METSCHNIKOFF, 1879) SOROKIN, 1883 (HYP.: CLAVICIPITACEAE)
}

\author{
S.O. Kassab ${ }^{1 *}$, E.S. Loureiro ${ }^{2}$, R.H. Barbosa ${ }^{1 * *}$, P.R.B. da Fonseca ${ }^{3 * * *}$, T.A. Mota ${ }^{1 *}$, C. Rossoni ${ }^{1 * *}$ \\ ${ }^{1}$ Universidade Federal da Grande Dourados, Faculdade de Ciências Biológicas e Ambientais, Rod. Dourados- \\ Itahum, km 12, CEP 79804-970, Dourados, MS, Brasil. E-mail: samirkassab@gmail.com
}

\section{RESUMO}

\begin{abstract}
Sugere-se uma alteração no método de amostragem da cigarrinha-da-raiz para a cultura da cana-de-açúcar com o objetivo de aprimorar o controle da praga. O monitoramento convencional de Mahanarva fimbriolata pode subestimar o número de ovos da área, ocasionando ressurgência da praga. Neste sentido, um estudo foi realizado em Ponta Porã, MS, com o objetivo de alterar a proposta de amostragem da M. fimbriolata. Odelineamento experimental composto por blocos casualizados, com dez tratamentos e quatro repetições. Os tratamentos testados foram: 1) Testemunha - não tratada, 2) Thiamethoxam 250WG - $250 \mathrm{~g} \mathrm{ha}^{-1}$, 3) IBCB 425 - $5 \times 10^{9} \mathrm{con} \mathrm{ha}^{-1}$, 4) IBCB 425 - 10 x $10^{9}$ con ha $^{-1}$, 5) IBCB $425-15 \times 10^{9}$ con ha $^{-1}$, 6) BIO $08-5 \times 10^{9}$ con ha- $^{-1}$, 7) BIO $08-10 \times 10^{9}$ con ha $^{-1}$, 8) BIO 08 - $15 \times 10^{9} \mathrm{con} \mathrm{ha}^{-1}$, 9) IBCB 425 - $5 \times 10^{9} \mathrm{con} \mathrm{ha}^{-1}$ - aplicação granulada e 10) BIO 08 - $5 \times 10^{9}$ con ha- ${ }^{-1}$ aplicação granulada. As avaliações de eficiência dos tratamentos obtidas aos 15, 45, 75 e 105 dias após a aplicação (DAA). Aos 15 DAA os tratamentos com os isolados BIO 08 - aplicação granulada e IBCB 425 nas doses de $5 \times 10^{9}$ con ha $^{-1}$ e $10 \times 10^{9}$ con ha $^{-1}$ apresentaram eficiência de 73,33 e 70,66\%, respectivamente. Não houve diferenças significativas entre os tratamentos com os inseticidas biológicos e químicos testados no experimento aos 75 e 105 DAA.
\end{abstract}

PALAVRAS-CHAVE: Cana-de-açúcar, cigarrinha-da-raiz, controle microbiano, fungos entomopatogênicos.

\begin{abstract}
CHANGE IN METHOD OF SAMPLING MAHANARVA FIMBRIOLATA (STÅL, 1854) (HEM.: CERCOPIDAE) AND EVALUATION OF EFFICIENCY OF METARHIZIUM ANISOPLIAE (METSCHNIKOFF, 1879) SOROKIN, 1883 (HYP.: CLAVICIPITACEAE). The present study suggests a change in the method of sampling the spittlebug in sugarcane crops in order to improve the control of this pest. Conventional monitoring of Mahanarva fimbriolata may underestimate the number of eggs in the area, causing pest resurgence. In this regard a study was carried out in Ponta Porã, MS, Brazil, in order to study possible changes in the methods used for sampling $M$. fimbriolata. The experiment consists of randomized blocks with 10 treatments and 4 replications. The treatments were: 1) control - untreated, 2) Thiamethoxam $250 \mathrm{WG}$ at $250 \mathrm{~g} \mathrm{ha}^{-1}$, 3) IBCB 425 at $5 \times 10^{9} \mathrm{con} \mathrm{ha}^{-1}$, 4) IBCB 425 at $10 \times 10^{9}$ con ha $^{-1}$, 5) IBCB 425 at $15 \times 10^{9}$ con ha $\left.^{-1}, 6\right)$ BIO 08 at $5 \times 10^{9}$ con ha $^{-1}$, 7) BIO 08 at $\left.10 \times 10^{9} \mathrm{con} \mathrm{ha}^{-1}, 8\right) \mathrm{BIO} 08$ at $15 \times 10^{9} \mathrm{con} \mathrm{ha}^{-1}$, 9) IBCB 425 at $5 \times 10^{9} \mathrm{con} \mathrm{ha}^{-1}$ - granular application and 10) BIO 08 at $5 \times 10^{9} \mathrm{con} \mathrm{ha}^{-1}-$ granular application. Evaluations of effectiveness of treatments were obtained at 15, 45, 75 and 105 days after application (DAA). At 15 DAA treatments with the isolated application with grainy BIO 08 and IBCB 425 at dosage of $5 \times 10^{9} \mathrm{con} \mathrm{ha}^{-1}$ and $10 \times 10^{9} \mathrm{con} \mathrm{ha}^{-1}$ showed an efficiency of 73.33 and $70.66 \%$, respectively. There were no significant differences between treatments with biological and chemical insecticides tested in the experiment at 75 and 105 DAA.
\end{abstract}

KEY WORDS: Entomopathogenic fungus, microbial control, sugar cane, spittlebug.

${ }^{2}$ Universidade Federal de Mato Grosso do Sul, Chapadão do Sul, MS, Brasil.

${ }^{3}$ Universidade Federal da Grande Dourados, Faculdade de Ciências Agrárias, Dourados, MS, Brasil.

*Doutorando em Entomologia e Conservação da Biodiversidade, Universidade Federal da Grande Dourados, Faculdade de Ciências Biológicas e Ambientais.

**Mestrando(a) em Entomologia e Conservação da Biodiversidade, Universidade Federal da Grande Dourados, Faculdade de Ciências Biológicas e Ambientais.

***Doutorando em Agronomia (Produção Vegetal), Universidade Federal da Grande Dourados, Faculdade de Ciências Agrárias. 
Mahanarva fimbriolata (Stål, 1854) (Hemiptera: Cercopidae) destaca-se como uma das principais pragas da cana-de-açúcar (BARBOSA et al., 2011). A mecanização da colheita das áreas destinadas ao cultivo da cana-de-açúcar proporcionou o acúmulo de palhada sobre o solo e os restos culturais, associados à elevada temperatura e umidade favoreceu o desenvolvimento das populações de $M$. fimbriolata (Dinardo-Miranda et al., 1999; DinARDO-Miranda et al.,2003; DinARDO-MIRANDA; GIL, 2007). Oaumento no número decigarrinhas promove perdas significativas na produtividade e qualidade da cana-de-açúcar (Dinardo-Miranda et al., 2000; DinARDo-Miranda et al., 2002; MADALENO et al., 2008).

Como a maioria das variedades de interesse comercial são suscetíveis às injúrias de ninfas e adultos de $M$. fimbriolata, pesquisas vêm sendo conduzidas com intuito de avaliar a eficiência de inseticidas eisolados do fungo Metarhizium anisopliae (METSCHNIKOFF, 1879) Sorokin, 1883 (Hypocreales: Clavicipitaceae) para o controle deste inseto (AlmEIDA et al., 2003; Loureiro et al., 2005; MENDONÇA, 2005; Almeida et al., 2007). Os estudos contribuíram para a implementação de um programa de controle da M. fimbriolata utilizando M. anisopliae e produtos fitossanitários químicos (DiNARDO-MirANDA et al., 2004).

O monitoramento de $M$. fimbriolata inicia-se após as primeiras chuvas do mês de setembro (ALMEIDA et al.,2007). As amostragens devem ser realizadas em 3 pontos por hectare em 2 metros lineares da linha de plantio ea periodicidadeideal para os levantamentos é de 15 dias (MendonçA, 2005; DinARDo-Miranda; GIL, 2007). A amostragem convencional desta praga considera as ninfas e adultos de $M$. fimbriolata e esta estratégia deve ser reelaborada.

Áreas com histórico de elevadas infestações de cigarrinhas fornecem dados amostrais inconsistentes, ou seja, nestes locais pode ter ocorrido elevada oviposição e a regularidade na precipitação pluviométrica ocasiona a ressurgência da praga comprometendo as aplicações de produtos fitossanitários para o controle de $M$. fimbriolata.

A proposta dealteraçãodo método deamostragem de $M$. fimbriolata reduz a possibilidade do produtor subestimar o potencial biótico (ovos diapáusicos e normais) da praga existente na área. Neste método propomos a amostragem de ninfas pequenas (até 5 $\mathrm{mm})$, médias (6-10 mm), grandes (acima de $10 \mathrm{~mm}$ ) e adultos (GAllo et al., 2002). A proposta de subdivisão das ninfas em pequenas, médias, grandes e adultos possibilita ao amostrador estimar o final de ciclo da geração de $M$. fimbriolata e a percepção do momento de aplicação do produto ocorre quando o número de ninfas grandes for maior que o de pequenas e médias. Neste período, adultos de $M$. fimbriolata também serão amostrados na população praga e isto pode indicar que a maioria dos ovos viáveis tiveram condições favoráveis para que as ninfas eclodissem e a possibilidade de ressurgência das populações de $M$. fimbriolata será menor.

O objetivo desta pesquisa foi propor uma alteração no método de amostragem da $M$. fimbriolata e observar a eficiência do fungo M. anisopliae.

O experimento foi conduzido, em um canavial da Usina BUNGE ${ }^{\circledR}$ - Unidade Monteverde AgroEnergética S/A, em Ponta Porã, Estado de Mato Grosso do Sul (MS) (latitude de $23^{\circ} 32^{\prime} 30^{\prime \prime} \mathrm{S}$ e a longitude de $55^{\circ} 37^{\prime} 30^{\prime \prime} \mathrm{O}$ ). A área experimental foi cultivada com a variedade SP81-3250 e a brotação da soqueira encontrava-se com 2 meses de idade. Utilizou-se o delineamento experimental em blocos ao acaso, composto por 10 tratamentos e quatro repetições, sendo cada unidade amostral representada por 10 sulcos de $10 \mathrm{~m}$ com espaçamento de 1,4 $\mathrm{m}$, totalizando $140 \mathrm{~m}^{2}$.

Os tratamentos utilizados foram compostos pela testemunha, dois isolados de M. anisopliae (IBCB 425 e BIO 08) e um inseticida químico, sendo estes: 1) Testemunha - não tratada, 2) Thiamethoxam 250WG - $250 \mathrm{~g} \mathrm{ha}^{-1}$, 3) IBCB 425 - $5 \times 10^{9}$ conídios (con) ha-1, 4) IBCB $425-10 \times 10^{9} \mathrm{con} \mathrm{ha}^{-1}$, 5) IBCB $425-15 \times 10^{9} \mathrm{con}$ $\mathrm{ha}^{-1}$, 6) BIO $08-5 \times 10^{9} \mathrm{con} \mathrm{ha}^{-1}$, 7) BIO $08-10 \times 10^{9} \mathrm{con}$ $\mathrm{ha}^{-1}$, 8) BIO 08 - $15 \times 10^{9} \mathrm{con} \mathrm{ha}^{-1}$, 9) IBCB 425 - $5 \times 10^{9}$ con ha- ${ }^{-1}$ - aplicação granulada e 10) BIO $08-5 \times 10^{9} \mathrm{con}$ ha $^{-1}$ - aplicação granulada. A aplicação das suspensões fúngicas foi realizada a partir da lavagem do arroz em águacontendoagentetensoativoa $0,1 \%$ nos tratamentos $3,4,5,6,7 \mathrm{e} 8$. Nos tratamentos $9 \mathrm{e} 10$ as aplicações foram a lance, ou seja, o arroz esporulado (conidiogênese + substrato) foi distribuído na área e no tratamento 2 efetuou-se aplicação do produto via líquida.

Para a instalação da pesquisa foram realizadas, semanalmente, amostragens de ninfas pequenas, médias, grandes e adultos de $M$. fimbriolata com o objetivo de identificar o momento de aplicação dos tratamentos, ou seja, final de ciclo da primeira geração de cigarrinhas. As avaliações de eficiência foram obtidas aos 15, 45, 75 e 105 dias após a aplicação (DAA), observando o número de ninfas vivas de $M$. fimbriolata.

A amostragem de ninfas foi em $2 \mathrm{~m}$ linear da linha de plantio, em um ponto por parcela. Os dados foram submetidos à análise de variância, após serem transformados em $\sqrt{x+0,5}$. A comparação das médias de ninfas por tratamento obtidas pelo teste de Duncan a 5\% de probabilidade e a eficiência dos produtos foram calculadas por meio da fórmula de Аввотт (1925).

Na amostragem prévia verificou-se que o número de ninfas de cigarrinhas, nas unidades amostrais, não variou entre os tratamentos. Nesta avaliação, a quantidade de ninfas médias e grandes havia aumentado e houve redução no número de ninfas pequenas. Além disso, pode-se observar que os 
adultos de $M$. fimbriolata também foram amostrados na população da praga (Tabela 1).

Na primeira avaliação (15 DAA), os tratamentos mais eficientes no controle das ninfas da $M$. fimbriolata foram obtidos pelos isolados BIO 08 com aplicação granulada e IBCB 425 nas doses de $5 \times 10^{9} \mathrm{con} \mathrm{ha}^{-1} \mathrm{e}$ $10 \times 10^{9}$ con ha ${ }^{-1}$, respectivamente (Fig. 1).

Ressalta-seque, nos tratamentos com os melhores resultados, a infestação de ninfas encontrava-se acima do nível de dano econômico conforme recomendado por DinARDO-MirANDA; GIL (2007). A proposta de avaliação leva em consideração o potencial biótico da área amostral, ou seja, os ovos normais e diapáusicos (viáveis). Como a aplicação ocorreu no final do primeiro ciclo de cigarrinhas, as demais gerações não alcançaram o nível de dano econômico proposto por DinARDO-MIRANDA; GIL (2007). Acreditamos que a maioria dos ovos normais e diapáusicos (viáveis) de $M$. fimbriolata teveram condições para a eclosão das ninfas e, assim, as chances de reaplicações diminuíram. Outra hipótese que justifica a eficiência obtida pelo fungo $M$. anisopliae na pesquisa, pode estar associada à época de aplicação do entomopatógeno, visto que as utilizações tardias de inseticidas reduziram as infestações de cigarrinhada-raíz, resultando em incrementos significativos na produtividade da cana-de-açúcar (DiNARDO-MIRANDA et al., 2004; MADALENO et al., 2008).

Tabela 1 - Média de ninfas e adultos de Mahanarva fimbriolata (Hemiptera: Cercopidae) por tratamento em cana-de-açúcar (SP81-3250) na amostragem prévia. Usina Bunge ${ }^{\circledast}$ - Unidade Monteverde Agro-Energética S/A, Ponta Porã, MS, 2010/2011.

\begin{tabular}{|c|c|c|c|c|}
\hline \multirow{2}{*}{ Tratamentos } & \multicolumn{4}{|c|}{ Amostragem prévia } \\
\hline & Ninfas pequenas & Ninfas médias & Ninfas grandes & Adultos \\
\hline Testemunha (não tratada) & 0 & 1,25 & 5,5 & 0,25 \\
\hline Thiamethoxam 250WG - $250 \mathrm{~g} \mathrm{ha}^{-1}$ & 0 & 1 & 2,5 & 0 \\
\hline IBCB $425\left(5 \times 10^{9}\right.$ con ha $\left.{ }^{-1}\right)$ & 0 & 1 & 4,25 & 0,5 \\
\hline IBCB $425\left(10 \times 10^{9}\right.$ con ha $\left.^{-1}\right)$ & 0 & 1 & 4 & 0 \\
\hline IBCB $425\left(15 \times 10^{9}\right.$ con ha-1 $\left.^{-1}\right)$ & 0,25 & 0 & 4,75 & 1 \\
\hline BIO $08\left(5 \times 10^{9}\right.$ con ha-1 $)$ & 0 & 0,75 & 6 & 0,25 \\
\hline BIO $08\left(10 \times 10^{9}\right.$ con ha $\left.^{-1}\right)$ & 0 & 1,5 & 2,25 & 0 \\
\hline BIO $08\left(15 \times 10^{9}\right.$ con ha $\left.^{-1}\right)$ & 0,75 & 1,5 & 1 & 0,25 \\
\hline IBCB $425\left(5 \times 10^{9}\right.$ con ha-1) aplicação granulada & 0,25 & 0,75 & 5,25 & 0,25 \\
\hline BIO 08 (5 x $10^{9}$ con ha-1) aplicação granulada & 0,25 & 1,75 & 4,25 & 1,2 \\
\hline
\end{tabular}

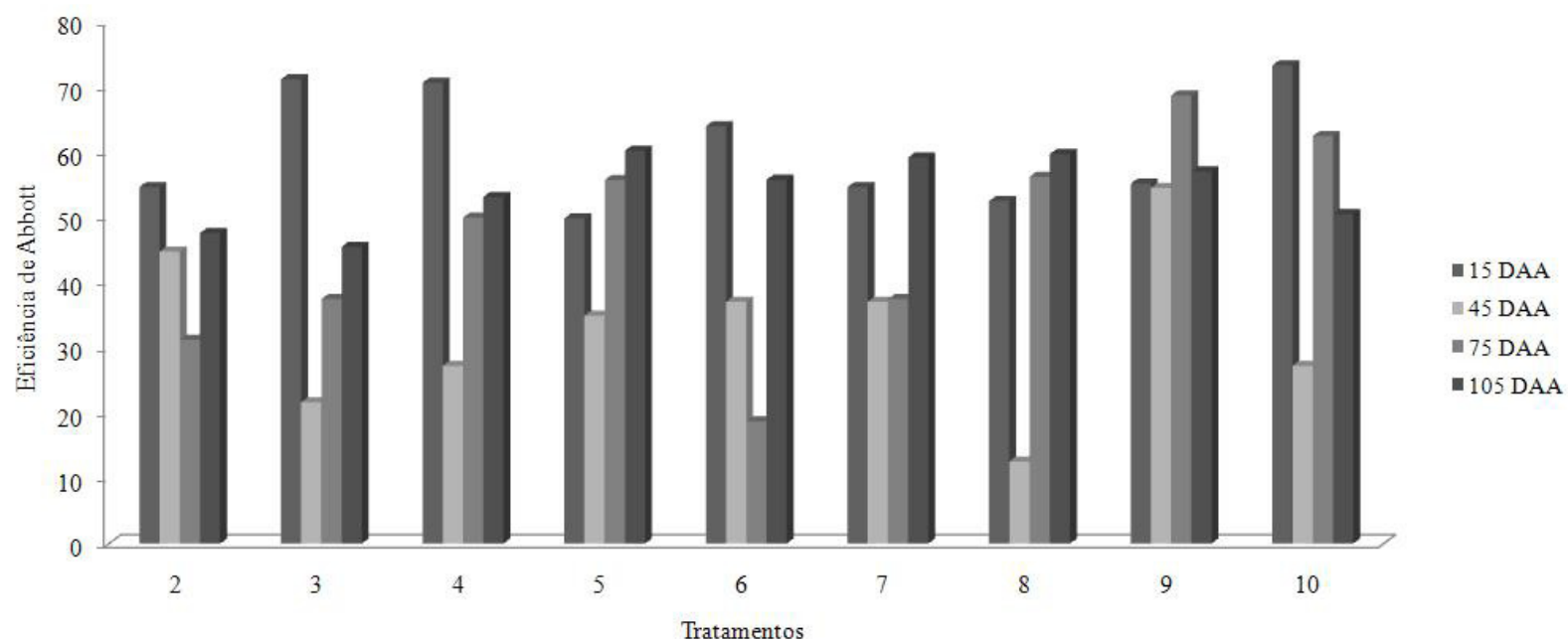

Fig. 1 - Eficiência dos diferentes tratamentos [2) Thiamethoxam 250WG - $250 \mathrm{~g} \mathrm{ha}^{-1}, 3$ ) IBCB $425-5 \times 10^{9} \mathrm{con} \mathrm{ha}^{-1}$, 4) IBCB 425 - $10 \times 10^{9}$ con ha $^{-1}$, 5) IBCB 425 - $15 \times 10^{9}$ con ha- $^{-1}$, 6) BIO 08 - $5 \times 10^{9}$ con ha $^{-1}$, 7) BIO 08 - $10 \times 10^{9}$ con ha- $^{-1}$, 8) BIO 08 $15 \times 10^{9} \mathrm{con} \mathrm{ha}^{-1}$, 9) IBCB 425 - $5 \times 10^{9} \mathrm{con} \mathrm{ha}^{-1}$ - aplicação granulada e 10) BIO 08 - $5 \times 10^{9} \mathrm{con} \mathrm{ha}^{-1}$ - aplicação granulada] para o controle de ninfas de Mahanarva fimbriolata (Hemiptera: Cercopidae). Usina BUNGE ${ }^{\circledR}$ Unidade Monteverde Agro-Energética S/A, Ponta Porã, MS, 2010/2011. 
Ressalta-se, ainda, que os fungos atuam no tegumento de $M$. fimbriolata e os insetos mortos são considerados o maior depósito de estruturas infectantes do entomopatógeno e isto auxilia no controle da ressurgência da população praga (Alves et al., 1998).

Nas avaliações obtidas aos 75e 105DAA não ocorreram diferenças significativas entre os tratamentos com inseticidas biológicos e químicos. Observa-se ainda que, mesmo nos tratamentos com dosagem de $15 \times 10^{9}$ con ha $^{-1}$, o número de insetos amostrados não diferiu estatisticamente entre as dosagens de $M$. aniosopliae avaliadas (Tabela 2).

A alteração no método de amostragem do inseto pode explicar os resultados obtidos no presente estudo, pois a percepção do momento de aplicação (final de ciclo da primeira geração de $M$. fimbriolata) do produto foi suficiente para manter a população de ninfas abaixo do nível de dano econômico proposto por DinARDO-Miranda; GIL (2007).

De maneira geral, recomenda-se que as amostragens das populações de $M$. fimbriolata sejam efetuadas no início das primeiras chuvas do mês de setembro (Almeida et al., 2007). As áreas com histórico de elevadas infestações de $M$. fimbriolata devem ser priorizadas nos programas de monitoramento desta praga. Além disso, o momento de aplicação dos produtos fitossanitários deve ser sincronizado com o final de ciclo da geração de cigarrinha e nas condições já citadas neste trabalho.

Dessa forma, as possibilidades de reaplicação de inseticidas biológicos e químicos serão menores, pois os ovos viáveis da área em que será efetuado o controle já obtiveram condições favoráveis para que as ninfas eclodissem e o potencial biótico deste local em proporcionar novas gerações da praga será menor.

\section{CONCLUSÕES}

O monitoramento de ninfas pequenas, médias, grandes e adultos de $M$. fimbriolata pode aprimorar o momento de aplicação do inseticida biológico para o controle da cigarrinha-da-raiz e isto diminui as perdas e custos do tratamento por hectare.

\section{AGRADECIMENTOS}

À Coordenação de Aperfeiçoamento de Pessoal de Nível Superior (CAPES) e ao Conselho Nacional de Desenvolvimento Científico e Tecnológico (CNPq). À Usina BUNGE ${ }^{\circledR}$ - Unidade Monteverde Agro-Energética S/A e ao engenheiro agrônomo Antonio Higino F. Pereira (Coordenador de Processos Agrícolas). À empresa Biotech Controle Biológico Ltda., ao diretor Abel Ferreira da Rocha Neto e ao engenheiro agrônomo Caê Alonso Ramos pelo auxílio técnico/científico nesta nova proposta de amostragem.

\section{REFERÊNCIAS}

ABBOTT, W.S.A. A Method of computing the effectiveness of an insecticide. Journal of Economic Entomology, v.18, n.3, p.265-266, 1925.

ALMEIDA, J.E.M.; BATISTA FILHO, A.; SANTOS, A.S. Avaliação do controle biológico de Mahanarva fimbriolata (Hemiptera: Cercopidae) com o fungo Metarhizium anisopliae em variedades de cana-de-açúcar e diferentes épocas de corte. Arquivos do Instituto Biológico, São Paulo, v.70, n.1, p.101-103, 2003.

Tabela 2 - Média ( \pm erro padrão da média) de ninfas de Mahanarva fimbriolata (Hemiptera: Cercopidae) em de cana-de-açúcar (SP81-3250) nos tratamentos com produtos biológicos e químico nos dias após a aplicação (DAA). Usina BUNGE $^{\circledR}$ - Unidade Monte Verde, Ponta Porã, MS, 2010/2011.

\begin{tabular}{|c|c|c|c|c|}
\hline Tratamentos & 15 DAA & 45 DAA & 75 DAA & 105 DAA \\
\hline Testemunha (não tratada) & $3,75 \pm 1,08 \mathrm{a}$ & $1,43 \pm 0,90 \mathrm{a}$ & $4,00 \pm 1,12 \mathrm{a}$ & $3,80 \pm 0,16 a$ \\
\hline Thiamethoxam 250WG (250 $\left.\mathrm{g} \mathrm{ha}^{-1}\right)$ & $1,70 \pm 0,28 \mathrm{bc}$ & $0,79 \pm 0,40 \mathrm{ab}$ & $2,75 \pm 0,83 b$ & $1,99 \pm 0,50 \mathrm{~b}$ \\
\hline IBCB $425\left(5 \times 10^{9}\right.$ con ha-1 $\left.^{-1}\right)$ & $1,08 \pm 0,25 b c$ & $1,12 \pm 0,48 \mathrm{ab}$ & $2,50 \pm 0,75 b$ & $2,07 \pm 0,09 b$ \\
\hline IBCB $425\left(10 \times 10^{9}\right.$ con ha-1 $)$ & $1,10 \pm 0,28 b c$ & $1,04 \pm 0,25 \mathrm{ab}$ & $2,00 \pm 0,70 \mathrm{~b}$ & $1,78 \pm 0,44 b$ \\
\hline IBCB $425\left(15 \times 10^{9}\right.$ con ha-1) & $1,88 \pm 0,40 b$ & $0,93 \pm 0,91 \mathrm{ab}$ & $1,77 \pm 0,80 \mathrm{~b}$ & $1,51 \pm 0,37 b$ \\
\hline BIO $08\left(5 \times 10^{9}\right.$ con ha-1 $)$ & $1,35 \pm 0,28 b c$ & $0,90 \pm 0,85 \mathrm{ab}$ & $3,25 \pm 0,85 b$ & $1,68 \pm 0,44 b$ \\
\hline BIO $08\left(10 \times 10^{9}\right.$ con ha $\left.^{-1}\right)$ & $1,70 \pm 0,62 b c$ & $0,90 \pm 0,85 \mathrm{ab}$ & $2,50 \pm 0,88 b$ & $1,55 \pm 0,50 \mathrm{~b}$ \\
\hline BIO $08\left(15 \times 10^{9}\right.$ con ha-1 $\left.^{-1}\right)$ & $1,78 \pm 0,28 \mathrm{bc}$ & $1,25 \pm 0,28 \mathrm{~b}$ & $1,75 \pm 0,47 b$ & $1,53 \pm 0,37 b$ \\
\hline IBCB $425\left(5 \times 10^{9}\right.$ con ha $\left.^{-1}\right)$ aplicação granulada & $1,68 \pm 0,28 b c$ & $0,65 \pm 0,55 \mathrm{~b}$ & $1,25 \pm 0,25 b$ & $1,63 \pm 0,38 b$ \\
\hline 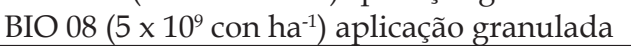 & $1,00 \pm 0,40 \mathrm{~b}$ & $1,04 \pm 0,28 \mathrm{ab}$ & $1,50 \pm 0,50 \mathrm{~b}$ & $1,88 \pm 0,14 b$ \\
\hline $\mathrm{CV}(\%)$ & 29,35 & 45,69 & 36,74 & 38,65 \\
\hline
\end{tabular}


ALMEIDA, J.E.M.; BATISTA FILHO, A.; DA COSTA, E.A.D. Efeito de adjuvantes em associação com Thiamethoxam 250 WG e Metarhizium anisopliae (Metsch.) Sorokin no controle de cigarrinha-da-raiz da cana-de-açúcar Mahanarva fimbriolata (Stal, 1854) (Hemiptera: Cercopidae). Arquivos do Instituto Biológico, São Paulo, v.74, n.2, p.135-140, 2007.

ALVES, S.B. Fungos entomopatogênicos. In: ALVES, S.B.(Ed.) Controle microbiano de insetos. 2.ed. Piracicaba: FEALQ, 1998. cap. 11, p.289-381.

BARBOSA, R.H.; KASSAB, S.O.; FONSECA, P.R.B.; ROSSONI, C.; SILVA, A.S. Associação de Metarhizium anisopliae (Hyp.: Clavicipitaceae) e thiamethoxam para o controle da cigarrinha-das-raízes em cana-de-açúcar. Ensaios e Ciência, v.15, n.5, p.41-51, 2011.

DINARDO-MIRANDA, L.L.; GIL, M.A. Estimativa do nível de dano econômico de Mahanarva fimbriolata (Stål) (Hemiptera: Cercopidae) em cana-de-açúcar. Bragantia, v.66, n.1, p.81-88, 2007.

DINARDO-MIRANDA, L.L.; FIGUEIREDO, P.; LANDELL, M.G.A.; FERREIRA, J.M.G.; CARVALHO,

P.A.M. Estimativa de danos causados pelas cigarrinhas das raízes (Mahanarva fimbriolata) a diversos genótipos de cana-de-açúcar. STAB: Açúcar, Álcool e Subprodutos, v.17, n.5, p.48-52, 1999.

DINARDO-MIRANDA, L.L.; FERREIRA, J.M.G.; CARVALHO, P.A.M. Influência das cigarrinhas das raízes, Mahanarva fimbriolata, sobre a qualidade tecnológica da cana-de-açúcar. STAB: Açúcar, Álcool e Subprodutos, v.19, n.3, p.34-35, 2000.

DINARDO-MIRANDA, L.L.; GARCIA, V.; PARAZZI, V.J. Efeito de inseticidas no controle de Mahanarva fimbriolata (Stål) (Hemiptera: Cercopidae) e de nematóides fitoparasitos na qualidade tecnológica e na produtivida- de da cana-de-açúcar. Neotropical Entomology, v.31, n.4, p.609-614, 2002.

DINARDO-MIRANDA, L.L.; NAKAMURA, G.; ZOTARELLI. L.; BRAZ, B.A.; EUZÉBIO, O. Viabilidade técnica e econômica de Actara 250 WG, aplicado em diversas doses, no controle de cigarrinha-das-raízes. STAB: Açúcar, Álcool e Subprodutos, v.22, n.1, p.39-43, 2003.

DINARDO-MIRANDA, L.L.; COELHO, A.L.; FERREIRA, J.M.G. Influência da época de aplicação de inseticidas no controle de Mahanarva fimbriolata (Stål) (Hemiptera: Cercopidae), na Qualidade e na Produtividade da Cana-de-açúcar. Neotropical Entomology, v.33, n.1, p.91-98, 2004.

GALLO, D.; NAKANO, O.; SILVEIRA NETO, S. CARVALHO, R.P.L.; BAPTISTA, G.C.; BERTI FILHO, E.; PARRA, J.R.P.; ZUCCHI, R.A.; ALVES, S.B.; VENDRAMIM, J.D.; MARCHINI, L.C.; LOPES, J.R.S.; OMOTO, C. Entomologia agrícola. Piracicaba: Fealq, 2002. 920p.

LOUREIRO, E.S.; BATISTA FILHO A.; ALMEIDA, J.E.M.; PESSOA, L.G.A. Seleção de isolados de Metarhizium anisopliae (Metch.) Sorok. contra a cigarrinha-da-raiz da cana-de-açúcar Mahanarva fimbriolata (Stål) (Hemiptera: Cercopidae) em laboratório. Neotropical Entomology, v.34, n.5, p.791-798, 2005.

MADALENO, L.L.; RAVANELI, G.R.; PRESOTTI, L.E.; MUTTON, M.A.; FERNANDES, O.A.; MUTTON, M.J.R. Influence of Mahanarva fimbriolata (Stål) (Hemiptera: Cercopidae) injury on the quality of cane juice. Neotropical Entomology, v.37, n.1, p.68-73, 2008.

MENDONÇA, A.F. (Ed.). Cigarrinhas da cana-de-açúcar: controle biológico. Maceió: Insecta, 2005. 317p.

Recebido em 18/4/11

Aceito em 19/10/12 